\title{
Análisis de las aulas de acogida lingüística: carácter inclusivo, flexibilidad e integración en la organización del centro
}

\author{
Analysis of linguistic reception classrooms: inclusive character, flexibility and \\ integration in the school organization
}

\author{
Raúl García Medina \\ romedina@ucm.es \\ Universidad Complutense de Madrid. España
}

\section{Resumen}

La proliferación en nuestro país de las denominadas aulas de acogida lingüística respondió a la inexistencia de medidas y recursos en los centros educativos para apoyar el aprendizaje de la lengua vehicular del alumnado extranjero de incorporación tardía. Se analizarán estos dispositivos desde la perspectiva de su organización y funcionamiento (en concreto, los implantados en Andalucía, Madrid y Cataluña), con el propósito de demostrar que no pueden considerarse agrupamientos flexibles ni están organizados a partir de criterios de inclusión educativa. En la mayoría de los casos, se aprecia una endeble integración en la organización general del centro y permanecen al margen de las decisiones pedagógicas y de la dinámica relacional.

Palabras clave: aulas lingüísticas; agrupamientos flexibles; educación inclusiva; organización del centro; lengua vehicular.

\begin{abstract}
The proliferation in our country of the so-called linguistic reception classrooms responded to the lack of measures and resources, in the educational centers, to support foreign students of late incorporation in the learning of the vehicular language. These devices will be analyzed from the perspective of their organization and operation (in particular those implemented in Andalusia, Madrid and Catalonia), in order to demonstrate that they cannot be considered flexible groupings nor are they organized based on educational inclusion criteria. In most cases, there is a weak integration in the general organization of the center and they remain outside the pedagogical decisions and relational dynamics.
\end{abstract}

Keywords: linguistic classrooms; flexible groupings; inclusive education; organization of the center; vehicular language.

Recibido / Received: 17-01-2018

Aceptado / Accepted: 02-03-2018

Cómo referenciar este artículo / How to reference this article:

García, R. (2018). Análisis de las aulas de acogida lingüística: carácter inclusivo, flexibilidad e integración en la organización del centro. Tendencias Pedagógicas, 32, 91-105. inicial-final. doi: 10.15366/tp2018.32.007 


\section{Introducción}

En lo que llevamos de siglo XXI, los agrupamientos de estudiantes, con diferentes formatos, han sido una de las medidas que los centros educativos españoles han empleado con mayor frecuencia para atender las necesidades derivadas de la diversidad cultural y lingüística.

En concreto, cuando se trata de alumnado cuya lengua de origen no es la misma que la empleada en el proceso de aprendizaje, la respuesta más común de las distintas administraciones educativas ha sido su escolarización en aulas específicas separadas, donde se centraban en la enseñanza de la lengua de instrucción de la escuela. El supuesto que subyace tras este modelo es que la inclusión del alumnado alófono debe depender de su dominio de la lengua vehicular, lo que justifica una segregación temporal.

Podemos encontrar diferentes programas de acogida o bienvenida al alumnado extranjero, dependiendo de la comunidad autónoma en la que nos encontremos. Generalmente, todos estos programas han incluido la organización de aulas específicas para atender al alumnado alófono, pero con normativas diferentes y un funcionamiento que conviene analizar para esclarecer, en la medida de lo posible, hasta qué punto comparten un mismo enfoque a la hora de atender la diversidad cultural.

El precedente de este tipo de aulas se encuentra en Montreal (Quebec), donde comenzaron a utilizarse en la década de los años 60 del pasado siglo, extendiéndose por todo el país a partir de 1977 (Mc Andrew, 2001). Se concibieron como aulas específicas para la enseñanza del francés, la lengua vehicular de la escuela, de manera que el alumnado inmigrante, tras permanecer un año en ellas, pudiera incorporarse al aula regular.

Considerando las frecuentes modificaciones que se han sucedido en su regulación y la disminución provocada por los recortes de la administración, que están abocando a la desaparición a algunas de ellas, la enumeración que encontramos en García Fernández et al. (2009) puede aportarnos una idea aproximada de la diversidad de modalidades y formatos que han adoptado estas aulas en nuestro país: Aulas Temporales de Adaptación Lingüística (ATAL) en Andalucía y Extremadura; Aulas de Acogida en Cataluña, Islas Baleares y Murcia; Aulas de Enlace en Madrid; Aulas Temporales de Inmersión Lingüística (ATIL) en Asturias; Aulas de Inmersión Lingüística (AIL) en Navarra y La Rioja; Aulas de Adaptación Lingüística y Social (Aulas ALISO) en CastillaLeón; Grupos de Adaptación de la Competencia Curricular y Grupos de Adquisición de las Lenguas en Galicia; Programas de Acogida al Sistema Educativo (PASE) en la Comunidad Valenciana; Programa de Refuerzo Lingüístico en el País Vasco.

Ante esta variedad de aulas conviene adoptar una denominación genérica para referirnos a todas ellas. Algunas opciones son: "aulas lingüísticas" (Grañeras et al., 2007), "aulas especiales" (Fernández Echeverría y García Castaño, 2015) o "aulas de acogida lingüística" (Siqués, Perera y Vila, 2012), entre otras. Quizá porque recuerda el principal compromiso que deberían asumir estas aulas, la acogida, se adoptará esta última designación en todo el texto para facilitar la lectura.

\section{Enfoque del análisis}

En los primeros años de funcionamiento de estas aulas se consideró que pese a su variedad compartían una serie de rasgos, principalmente "su carácter intermedio, abierto, intensivo y flexible" (Grañeras et al., 2007: 163). Sin embargo, Goenechea, García y Jiménez (2011), al comparar el funcionamiento de las aulas implantadas en Andalucía y en Madrid, concluyen que ninguna de estas dos modalidades se corresponde exactamente con esa caracterización. Ponen en duda la apertura de las Aulas de Enlace madrileñas o el carácter intensivo de las Aulas Temporales de Inmersión Lingüística andaluzas. No obstante, la revisión que se presenta a continuación únicamente se centrará en el cuestionamiento de la flexibilidad de estos dispositivos, ya que las demás peculiaridades pueden inferirse a partir de la determinación del grado de flexibilidad en su 
organización.

Por otra parte, conviene precisar que se adoptará una perspectiva eminentemente organizativa en el análisis de estas aulas, atendiendo también a su posible implantación dentro de una iniciativa o programa más amplio para atender la diversidad cultural. Aunque no se aborden con detenimiento otras características o variables como la integración de las enseñanzas impartidas en el currículo obligatorio, la formación del profesorado, la metodología adoptada para la enseñanza de segundas lenguas o los materiales didácticos empleados, sí que se podrán encontrar algunas referencias a estas cuestiones, en la medida que sean necesarias para explicar el sentido de la implantación y organización de estos dispositivos. No obstante, las categorías fundamentales que se tomarán como referencia para establecer las conclusiones del análisis serán: los criterios que justifican este tipo de agrupamiento y su flexibilidad; el grado de integración en el funcionamiento general del centro; por derivación, su funcionamiento congruente con los principios de la educación inclusiva.

Con respecto a la primera de las categorías, es necesario recordar lo que tradicionalmente se ha entendido por agrupamientos flexibles en el ámbito de la Didáctica y de la Organización Escolar. En una obra ya clásica, como la de Antúnez y Gairín (1994), se caracterizan como aquellos agrupamientos no permanentes, organizados en función de la materia o actividad académica, que permiten respetar el ritmo de aprendizaje del alumnado. Lo autores hacen referencia a una división del grupo-clase en unidades más pequeñas que también admite la incorporación de estudiantes de otros grupos-clase del mismo nivel (agrupamiento horizontal), o de diferentes niveles (agrupamiento vertical). Los pequeños grupos formados deben tener un tamaño razonable y adecuado a la tarea, reunir estudiantes con alguna capacidad o habilidad semejante, favorecer la interacción entre el alumnado, implicar la utilización de estrategias metodológicas variadas y facilitar que el alumnado pueda adscribirse voluntariamente al grupo.

Por su parte, Molina (1995) señala las ventajas de los agrupamientos flexibles frente a los fijos y homogéneos, supeditando su organización a los objetivos que se persigan y a la naturaleza de las actividades educativas, de manera que pueda alcanzarse la mejor adaptación posible a las distintas situaciones de aprendizaje. En las dos fuentes citadas no se especifica con claridad quiénes deben ser los profesionales responsables de la organización de este tipo de agrupamientos, aunque en todo momento se sobreentiende que se trata de decisiones pedagógicas tomadas en el marco del grupoaula y del centro educativo.

Sin embargo, la ligereza con la que se han venido empleado los agrupamientos flexibles para atender a la diversidad del alumnado, requiere de una reflexión detenida, juiciosa $y$, fundamentalmente, didáctica. En este mismo sentido se manifiesta Oliver (2010) al proponer los criterios que deben orientar la organización de estos agrupamientos, y que se resumen a continuación:

- Deben ser heterogéneos en cuanto a su composición.

- Requieren un cambio en la organización del centro, de manera que se garantice la cooperación de los equipos docentes, tanto los que participan en la organización del agrupamiento como los que no.

- Es necesaria una reelaboración curricular, metodológica y una disposición de los recursos, medios y materiales didácticos adecuados a las necesidades del alumnado participante y de los grupos establecidos.

- No pueden organizarse sin contar con el deseo de participar de todos los implicados (alumnado, profesorado, familias, etc.) y sin el consenso de la comunidad educativa.

- Es prioritario tomar como referencia de todo proceso educativo al alumnado y su diversidad, potenciando sus capacidades y evitando cualquier peligro de marginación o segregación.

- Ha de establecerse un seguimiento educativo y psicológico que requiere un ejercicio consecuente de la tutoría. Manteniendo, en todo momento, el grupo-clase como referente para el alumnado. 
Advierte la autora que los agrupamientos flexibles no tendrán una positiva incidencia en el aprendizaje si dejan de ser potencialmente motivadores y diversificados. Sin duda, estas aclaraciones previas permitirán al lector apreciar con más claridad la trascendencia, implicaciones y limitaciones de las aulas de acogida lingüística que se describen con más detalle a continuación.

En cuanto al grado de integración en el funcionamiento general del centro, la segunda de las categorías de análisis, hay que tener en cuenta los márgenes que concede la normativa reguladora para que los centros educativos implanten estas aulas. Si se establecen instrucciones muy rígidas, que apenas dejen margen a la decisión de los centros, se corre el riesgo de que el centro implante el aula de acogida lingüística como una imposición de la administración, sin plantearse qué tiene que ver este dispositivo con el funcionamiento de los restantes órganos, estructuras y recursos del centro. En este sentido, Escudero (2016) presenta diversas investigaciones que ponen de manifiesto las dificultades para desarrollar los programas específicos o extraordinarios de atención a la diversidad, propuestos por la administración, cuando los centros educativos no acometen un proceso de transformación de su organización y prácticas educativas con la intención de acomodar dicho programa en su funcionamiento cotidiano.

Por tanto, será necesario prestar atención a algunos de los factores que pueden aportar información sobre el interés del centro educativo por integrar estas aulas en su funcionamiento general: la ubicación de las aulas, la coordinación del profesorado de las mismas con el resto de profesionales, la modificación de horarios para acomodar la actividad desarrollada en ellas, el replanteamiento del Proyecto Educativo y demás documentos programáticos del centro, etc.

Respecto a la tercera de las categorías de análisis, se tomará como referencia la concepción que ofrece Ainscow de la inclusión educativa "como un proceso de incremento de la participación de los alumnos en las culturas, currículos y comunidades de sus escuelas locales y de reducción de su exclusión de los mismos" (2001, p. 293). Atendiendo a los principios educativos que se derivan del planteamiento de Ainscow, tal y como lo recogen Echeita y Cuevas (2011), es fundamental analizar la consideración otorgada en estas aulas a las necesidades educativas del alumnado y a su diversidad lingüística y cultural. Todo lo cual, nos lleva a prestar atención a: la organización, evitando la segregación y la exclusión, de los apoyos educativos; la implicación de toda la comunidad educativa en la lucha contra la discriminación y el reconocimiento de la diversidad como un valor; la corresponsabilidad de todo el equipo de profesores en la atención a la diversidad; la revisión del currículo de la escuela para ajustarlo a la diversidad y asegurar su atención; por último, los apoyos con que cuenta el profesorado para atender las necesidades educativas de todo el alumnado. Sin duda, algunos de estos factores están íntimamente relacionados, y en coherencia, con los señalados en las dos anteriores categorías de análisis.

Por último, dada la variedad de aulas de acogida lingüística aparecidas en nuestro país, se ha preferido circunscribir este análisis, para no alargarlo en exceso, a tres de ellas, seguramente las que han sido objeto de más estudios y han suscitado una mayor producción científica. Nos referimos a las aulas implantadas en las comunidades autónomas de Andalucía, Cataluña y Madrid. La descripción, ofrecida a continuación, de estas tres modalidades de aulas está basada en el análisis de la normativa que las regula y la revisión de algunas de las investigaciones más relevantes sobre su implantación y funcionamiento. Con bastante probabilidad, estos tres casos aportarán una imagen bastante aproximada de lo que son este tipo de aulas, su articulación en la organización de los centros educativos y los objetivos que pretenden alcanzar.

3. Descripción y análisis del funcionamiento de las aulas de acogida lingüística: los casos de Andalucía, Cataluña y Madrid

\subsection{Las Aulas Temporales de Adaptación Lingïistica en Andalucía}

Andalucía fue la primera comunidad autónoma que implantó un dispositivo de este tipo, las denominadas Aulas Temporales de Adaptación Lingüística (en adelante ATAL). Concretamente en la provincia de Almería, en el contexto de una experiencia piloto desarrollada por dos centros 
educativos, se implantaron las Aulas Puente, su primera denominación, en 1997 (Ortiz, 2006). La Delegación Provincial de Educación y Ciencia de Almería promulgó las primeras instrucciones de funcionamiento en el año 2000, pero el reconocimiento oficial se produjo gracias al Plan para la Atención Educativa del Alumnado Inmigrante (2001), en el que, con su actual denominación de ATAL, se las considera una "medida específica" de apoyo al aprendizaje de la lengua española. Posteriormente, estas aulas se extendieron al resto de provincias andaluzas.

Un análisis de la normativa reguladora (Orden de 15 de enero de 2007) pone de manifiesto que la decisión sobre la creación y ubicación de las ATAL es competencia de las diferentes delegaciones provinciales de educación, contando con la información proporcionada por los centros y con un estudio de la zona emitido por los Equipos de Orientación Educativa y el servicio de Inspección. En todo caso, su ubicación no es definitiva, se revisa todos los cursos en función de las nuevas necesidades.

Como señala Ortiz (2006), en sus comienzos las ATAL fueron aulas permanentes o fijas, ubicadas en un centro concreto con un elevado índice de alumnado inmigrante. Estas aulas fijas eran de ámbito zonal y atendían a estudiantes de otros centros próximos que no disponían de ellas. Además, el profesorado de estas aulas tenía dedicación exclusiva y formaba parte del claustro de profesores y profesoras del centro. Fue más tarde cuando se introdujo una modalidad itinerante de ATAL, cuya característica principal era que el profesor se desplazaba para atender varios colegios de una misma localidad o de localidades cercanas. Iglesias y Goenechea (2016) constatan la existencia de un mayor número de aulas itinerantes (salvo en Almería, que están a la par con las fijas), atendiendo a los datos obtenidos en dos investigaciones, 2007 (Jiménez et al., 2009) y 2015, realizadas en la provincia de Cádiz, en las que se recaba la opinión de diversos profesionales y se desarrollan varios estudios de caso. En estas investigaciones se revelan los numerosos inconvenientes que ve el profesorado de las ATAL en las aulas itinerantes, entre otros la escasa integración del profesorado de estas aulas en el claustro (en lo que coinciden con Castilla, 2011), pero sobre todo lo complicado que resulta, en la práctica, ofrecer el apoyo dentro del aula, a pesar de que así está establecido en la normativa. Sin duda, estas evidencias cuestionan tanto la flexibilidad del recurso como su integración en el funcionamiento del centro. Según las conclusiones de Iglesias y Goenechea (2016) las ATAL fijas, utilizadas adecuadamente, sí que posibilitan el apoyo dentro del aula, por lo que podría considerarse un modelo más inclusivo que el que representan las aulas itinerantes.

El cometido de las ATAL es la atención al alumnado con escaso nivel de competencia lingüística en español, escolarizado en el segundo y tercer ciclos de Primaria o en la Educación Secundaria Obligatoria. Este alumnado se incorpora al ATAL a petición del tutor o tutora del aula ordinaria, emprendiéndose un proceso de evaluación inicial (se utiliza un modelo relativamente normalizado) que permita al profesorado del ATAL elaborar una programación de aula, para incluirla en el Proyecto de Etapa, y los consecuentes informes individualizados de evaluación trimestrales. No obstante, las instrucciones han ido variando en lo que a los criterios de selección del alumnado se refiere. Al respecto, Castilla (2011) advierte de la imposibilidad de referirse a un único y definido perfil de alumnado, ya que la realidad en algunos centros es que se atiende a estudiantes que no obedecen a las características fijadas en la normativa. Además, continúa diciendo, existen dudas sobre la univocidad y equivalencia de las evaluaciones a la hora de establecer el nivel de dominio del castellano del alumnado de las ATAL. El hecho de que no exista una clara identificación de las necesidades educativas del alumnado pone en entredicho que estos dispositivos puedan ofrecer una respuesta ajustada y coherente con los principios de la educación inclusiva.

En cuanto a la duración de la atención recibida, la normativa establece que será un máximo de 3 meses para las ATAL fijas en Educación Primaria y de un curso para las ATAL fijas de Educación Secundaria, así como para todas las aulas itinerantes en ambas etapas. Pero se contemplan algunas excepciones (enfermedad, absentismo, escolarización irregular en el país de origen, dificultades de aprendizaje, etc.) que podrían justificar la permanencia del alumno o alumna el doble del tiempo inicialmente permitido. No obstante, Castilla (2011), señala la existencia de numerosos casos en los que se excede el tiempo de estancia fijado por la normativa. La explicación, que ofrecen la mayoría 
de las investigaciones a este hecho, es que el profesorado de las aulas ordinarias prefiere que este alumnado permanezca el mayor tiempo posible en el aula específica, para evitar que su escaso dominio de la lengua perturbe los procesos de enseñanza y aprendizaje. Que la normativa fije el tiempo de estancia en estas aulas no dice mucho en favor de su flexibilidad, a lo que hay que unir la falta de coordinación entre el profesorado y el sentido utilitarista que se le da a los apoyos educativos (más centrado en facilitar la labor del profesorado que en atender las verdaderas necesidades del alumnado), lo que demuestra que se trata de una medida segregadora y excluyente.

Un aspecto importante, en relación con la organización y funcionamiento de las ATAL, es que se limita a 12 el número máximo de estudiantes que pueden ser atendidos. Asimismo, su asistencia deberá organizarse de manera que puedan regresar al aula ordinaria cuando se estén impartiendo asignaturas como Educación Física, Música, Educación Plástica y Visual o cualquier otra que no exija un dominio del idioma determinante para el acceso al currículo ordinario. Hay que añadir, como ya se señaló anteriormente, que la normativa prioriza la atención dentro del aula ordinaria, siempre que sea posible; tan solo se debe organizar el apoyo fuera del aula cuando existan especiales dificultades en la comprensión y expresión en lengua castellana. Es llamativo que la normativa establezca este criterio, que si se aplicara con el máximo rigor podría poner en cuestión la misma existencia de estas aulas. Volveremos sobre este asunto más adelante, pues resulta clave a la hora de enjuiciar la conveniencia de este tipo de dispositivos.

\subsection{Las Aulas de Enlace en Madrid}

Las primeras Aulas de Enlace aparecieron en el curso 2002-2003 como un programa experimental, regulándose su funcionamiento gracias a las Instrucciones de comienzo del curso 2003-2004. En el curso 2004-2005 pasaron a considerarse una medida estable y asumida, sin que se hayan publicado evaluaciones oficiales durante esos años que pudieran justificar su consolidación y generalización. La última normativa que se publicó, vigente en la actualidad, fueron las Instrucciones de 28 de julio de 2008 de la Viceconsejería de Educación de la Comunidad de Madrid (2008), justo antes del inicio del curso en el que se registró por última vez un incremento del número de Aulas de Enlace, aunque ya muy atenuado (García, Moreno y Salguero, 2014; García, 2015). En un primer momento, las Aulas de Enlace despertaron ciertas expectativas, como se puede apreciar en algunos estudios (Núñez y Cerrillo, 2004; Quicios y Miranda, 2005; del Olmo, 2012), que pronto se verían defraudadas.

Les corresponde a las diferentes direcciones de área territorial, en las que se subdivide la administración educativa de la Comunidad de Madrid, la responsabilidad de proponer la apertura de un Aula de Enlace, siempre que así lo acepte el centro educativo elegido. El problema es que, al considerarse una medida para dar servicio a una zona geográfica determinada, los centros cercanos con una población de alumnado alófono considerable ya no pueden disponer de Aula de Enlace, de manera que este alumnado tendrá que abandonar su colegio o instituto para incorporarse a aquel que sí cuente con el dispositivo.

El objetivo principal de las Aulas de Enlace ha sufrido alguna modificación. Mientras en las primeras instrucciones se establecía que debían atender al alumnado que desconociera la lengua española o con graves carencias en conocimientos básicos, en las instrucciones de 2008 se especifica que únicamente atenderán al alumnado procedente de un sistema educativo extranjero que desconozca la lengua española. Posiblemente el cambio se deba a las numerosas críticas (Martín y Mijares, 2007; Inglés, 2009; García et al., 2009, 2010, 2011; Sánchez y García, 2011; García y Moreno, 2014; García, 2015) que suscitó la posibilidad de que alumnado extranjero hispanohablante con cierto desfase curricular pudiera asistir a estas aulas, lo que lamentablemente ocurría en muchos centros. En las instrucciones de 2008 se puso especial cuidado en diferenciar el caso del alumnado extranjero con conocimiento de la lengua española que, en todo caso, podrá acceder a medidas de refuerzo educativo si existe ese desfase curricular, y no a un Aula de Enlace.

Igual que en el caso de las ATAL, las Aulas de Enlace atienden al alumnado del segundo y tercer ciclo de Educación Primaria o de Educación Secundaria Obligatoria, sin superar los 12 estudiantes 
(también se especifica que no podrán ser menos de 5). Sin embargo, no se estableció el mismo periodo máximo de permanencia, pues en el caso de las Aulas de Enlace es de 9 meses que pueden tanto acortarse, si el alumnado adquiere antes un dominio adecuado del español, como ampliarse, siempre con la autorización de la Dirección de Área Territorial y el informe favorable del Servicio de Inspección Educativa. Una de las características de las Aulas de Enlace, que contrasta con otras aulas de acogida lingüística, es que cuando se agota el periodo máximo de estancia se determina la incorporación del alumnado al grupo ordinario de referencia en el mismo centro escolar, siempre que sea posible. Lo que deja un resquicio a la posibilidad, como critican las investigaciones ya citadas, de que este alumnado se incorpore a un aula ordinaría de otro centro escolar diferente, el que determine la Comisión de Escolarización. No parece que nos encontremos ante un agrupamiento flexible cuando se trasciende, de manera tan evidente, los límites del centro educativo, descontextualizando la enseñanza y poniendo en riesgo la inclusión del alumnado.

En cuanto al tiempo de permanencia en el Aula de Enlace, la normativa únicamente especifica que el alumnado se incorporará a su grupo de referencia lo antes posible en aquellas áreas o materias que faciliten su integración como, por ejemplo, Educación física o Educación artística. Es decir, que podría permanecer en el Aula de Enlace durante un tiempo indefinido, no superior a 9 meses, toda la jornada escolar, lo que desafortunadamente ha sido bastante habitual. En este sentido, las Aulas de Enlace someten al alumnado a un alejamiento de las aulas ordinarias mucho más severo que las ATAL.

Por otra parte, llama la atención que en las últimas instrucciones de 2008 haya desaparecido el compromiso explícito de ofrecer la formación necesaria al profesorado destinado en estas aulas, que sí existía en las primeras instrucciones y sucesivas. En este mismo sentido, también se modificó la dotación de dos profesores por aula a tiempo completo (uno y medio en Primaria), siempre en comisión de servicios, dejando a la consideración y compromiso de los centros la continuidad del recurso: "se fomentará la implicación de los profesores del centro en las Aulas de Enlace pudiéndoseles asignar horas de dedicación a las mismas. Un profesor de los que atienda el Aula de Enlace hará las funciones de tutor del grupo" (Comunidad de Madrid, 2008, p. 5). Parece demasiado evidente la pretensión de mantener este dispositivo a coste cero, sin dotar al centro del profesorado necesario; probablemente se trate de otra de las razones que podrían explicar que haya disminuido tanto, casi hasta desaparecer, el número de Aulas de Enlace en los centros públicos. En la actualidad, es algo mayor el número de ellas que se mantiene en centros concertados, a pesar de que estos escolarizan a menos del 18\% del alumnado extranjero en la Comunidad de Madrid (según datos del curso 2013-14, García, 2015).

Y es que, como demuestran García, Olmos y Ouafaa (2015), tras analizar los datos sobre migración en los últimos años, es muy difícil justificar la reducción de las ATAL y de las Aulas de Enlace (en coincidencia con García, Moreno y Salguero, 2014) alegando la disminución de la población extranjera, incluso si se considera el retorno de inmigrantes a sus países de origen. Los autores se inclinan a pensar que los diferentes gobiernos autonómicos se encontraron con el dilema de justificar ante sus votantes el mantenimiento de los recursos de apoyo al alumnado extranjero, en un momento de crisis económica y recortes en educación. La decisión ofreció pocas dudas, "primero para los de aquí..." como defendieron algunos partidos políticos, azuzando la xenofobia.

Teniendo en cuenta la demanda de apoyos y recursos para que el profesorado pueda atender las necesidades de todo el alumnado, es evidente que estas políticas educativas no favorecen la inclusión.

\subsection{Las Aulas de Acogida en Cataluña}

A diferencia de lo que ocurre en las dos anteriores comunidades autónomas, en Cataluña se da la circunstancia de que la práctica totalidad del alumnado inmigrante desconoce el catalán, la lengua de enseñanza de la escuela. Esto explica la preocupación del gobierno autonómico por desarrollar medidas específicas de apoyo lingüístico al alumnado inmigrante. Así, en 1999 se promulga el "Pla d'actuació per a la incorporació tardana d'alumnes", que preveé la implantación de los "Tallers de 
Llengua" en Primaria y los "Tallers d'Adaptació Escolar i Aprenentatges Instrumentals Basics" (TAE) en Secundaria. Según Siqués, Perera y Vila (2012), los TAE se correspondían con el modelo de aulas cerradas, donde la única preocupación era la enseñanza de la lengua de la escuela. Aunque posteriormente se modificó la regulación para disminuir las horas que el alumnado inmigrante pasaba en los talleres, nunca se procedió a una evaluación institucional.

Tras un cambio de gobierno el Departamento de Educación de la Generalitat de Catalunya dictó en 2004 el plan "Llengua, Interculturalitat i Cohesió Social" -LIC- (Departament d'Educació, 2004), fundado sobre los principios del plurilingüismo y la interculturalidad. Su objetivo principal era priorizar la acogida y la adaptación escolar del alumnado inmigrante, de manera que el dominio de la lengua escolar se derivara de su implicación en las interacciones sociales y en las actividades curriculares. Además de otras medidas, como el centro educativo inclusivo y el entorno escolar educador, el Plan LIC creó las Aulas de Acogida para el alumnado extranjero de incorporación tardía, con el objetivo de apoyar tanto su adaptación escolar como su desarrollo emocional, considerando las consecuencias de un proceso migratorio, y su progreso lingüístico (Besalú, 2006, ofrece un concienzudo análisis del Plan LIC). A diferencia de los TAE, se han realizado evaluaciones de las Aulas de Acogida desde su implantación con resultados positivos (entre otras: Vila, Perera y Serra, 2006; Vila et al., 2007, 2009; Vila et al., 2008; o Siqués, Perera y Vila, 2012), algunos de los cuales se exponen en el siguiente apartado.

Por regla general, la administración otorga la dotación de un tutor de Aula de Acogida a aquellos centros que tienen más de 9 estudiantes extranjeros de incorporación tardía, aunque también se barajan otros criterios como la disponibilidad presupuestaria o las características del centro. Además, el centro recibe otros recursos: materiales didácticos, orientaciones para la organización del apoyo y formación a los profesionales que intervienen en la acogida, especialmente, a los tutores de estas aulas.

En cuanto a su funcionamiento, se establece que el Aula de Acogida debe ser un aula abierta dentro del contexto de una escuela inclusiva-, flexible -adaptándose a las necesidades de aprendizaje y acogida del alumnado atendido-, y dinámica -como un elemento más de la acción educativa del centro ha de estar presente en los procesos de reflexión pedagógica habituales.

Lo destacable es que se prioriza la dotación de materiales, recursos y profesionales para atender al alumnado extranjero de incorporación tardía, permitiendo que sea el propio centro el que tome la decisión sobre la organización y funcionamiento del Aula de Acogida, en función de las necesidades del alumnado concreto que escolariza y de su idiosincrasia organizativa y pedagógica. Por tanto, el centro podrá optar por organizar diferentes agrupamientos, según criterios como la escolarización previa, la lengua de origen o cualquier otra característica del alumnado, o decidirse por permitir que el tutor del Aula de Acogida ofrezca el apoyo en el aula ordinaria, organizando grupos de trabajo heterogéneos (también se admite combinar ambas modalidades). Todo lo cual, responde de manera más nítida a los principios de la educación inclusiva, además de establecer las condiciones para que desde el centro se organicen verdaderos agrupamientos flexibles.

En el caso de que se prefiera organizar un Aula de Acogida que ocupe su propio espacio físico, fuera del aula ordinaria, los grupos deberán ser reducidos -no más de 12 estudiantes-y el alumnado no podrá permanecer todo el horario lectivo en ella. Desde el momento de su escolarización se incorporarán, junto con sus compañeros del aula ordinaria, a las materias y actividades en las que el desconocimiento de la lengua vehicular no suponga una dificultad insalvable. Todo ello con el objetivo de asegurar una interacción intensa con el alumnado autóctono que facilite los procesos de socialización y aprendizaje activo de la lengua vehicular. En consecuencia, el alumnado no podrá recibir el apoyo en el Aula de Acogida durante más de 12 horas semanales ni permanecer en ella más allá de los 24 meses, o 36 en el caso de que provengan de culturas muy diferentes.

Es reseñable que la normativa insista en la importancia de que sea el centro educativo, en su totalidad, el principal responsable de la acogida. Por eso, y por el papel destacado que juega en el proceso de adaptación de este alumnado y su aprendizaje de la lengua vehicular, se recomienda que 
el tutor del Aula de Acogida sea un docente que tenga destino definitivo en ese centro y pertenezca al claustro; además de tener experiencia en enseñanza de lenguas y dominio de las tecnologías. Todas estas disposiciones parecen pretender que quede asegurado el proceso de inclusión del alumnado, aun cuando el centro se decante por implantar estas aulas. En contraste con los dos tipos de aulas anteriores, se trata de un modelo menos excluyente.

A pesar de que las numerosas evaluaciones de las Aulas de Acogida señalan avances significativos en la atención al alumnado extranjero de incorporación tardía, el Departamento de Educación de la Generalitat de Catalunya está proyectando su reconversión en unas nuevas "Aulas de Integración Social", con la intención de fomentar que el alumnado recién llegado pueda continuar sus estudios post-obligatorios, para lo que se pone el foco de atención en el acceso al lenguaje académico y al currículum ordinario (El Diari de l’Educació, 2015).

\section{Resultados relevantes de la investigación sobre las aulas de acogida lingüística}

Considerando la anterior descripción de algunas de las aulas de acogida lingüística, se puede establecer una primera y decisiva diferenciación, la que señalan Siqués, Perera y Vila (2012) al distinguir entre aulas cerradas, al margen de las aulas ordinarias y especializadas en la enseñanza de la lengua vehicular, y las aulas abiertas, asociadas al funcionamiento del aula ordinaria para ofrecer distintas posibilidades de atención que combinen el aprendizaje de la lengua vehicular con la inclusión y el aprendizaje en el aula ordinaria. Revisaremos más adelante los estudios sobre las ventajas de las aulas abiertas, pero antes conviene centrarse en el análisis de la modalidad más frecuente en nuestras escuelas, las aulas cerradas.

En efecto, aunque existan algunas diferencias referidas al grado de apertura o la extensión e intensidad del apoyo, parece evidente que tanto las ATAL como las Aulas de Enlace, y la mayoría de las aulas de acogida lingüística implantadas en nuestro país (por ejemplo, las aulas ALISO en Castilla-León, Arroyo, 2014), han sido concebidas como aulas cerradas y especializadas. Gran parte de las críticas realizadas a esta modalidad de apoyo lingüístico se refieren, precisamente, a su carácter cerrado y aislado del funcionamiento cotidiano del centro educativo. Resulta de interés, por tanto, presentar una recopilación general de los inconvenientes que señalan buena parte de las investigaciones sobre estos dispositivos. Para ello, tomaremos como referencia la enumeración presentada por García y Moreno (2014), aunque ampliando algunos aspectos:

- La segregación del alumnado en grupos homogéneos, conformados en virtud de características cognitivas, étnicas, lingüísticas o de cualquier otra índole, produce desigualdad, no favorece la cohesión social ni garantiza el aprendizaje, como se ha demostrado contundentemente en multitud de investigaciones (Franzé, 2008; Gibson y Carrasco, 2010; Martín y Mijares, 2007; INCLUD-ED, 2011). De las tres modalidades estudiadas, las Aulas de Enlace contribuyen, en mayor medida, a la segregación del alumnado, aunque las otras dos también se basan en grupos homogéneos.

- La notoria precipitación (en algunos casos, improvisación) con la que se implantaron este tipo de aulas, ante la evidencia de que el sistema educativo no estaba preparado para atender al alumnado extranjero y alófono (en Andalucía: Goenechea, García y Jiménez, 2011; Fernández Echeverría y García Castaño, 2015; en Castilla-León: Arroyo González, 2014; en Aragón: Navarro Sierra, 2003; en Madrid: Martín y Mijares, 2007; García et al., 2009, 2010, 2011; Sánchez y García, 2011).

- No responden a las necesidades de socialización del alumnado que ingresa tardíamente en el centro, poniendo en riesgo su inclusión educativa (Bonal, 2004; Roca, Úcar y Massot, 2002; Jiménez, 2004; García et al., 2009, 2010, 2011; Sánchez y García, 2011). La segregación en aulas separadas ocasiona que el proceso de inclusión quede en suspenso hasta la plena incorporación del alumnado al aula ordinaria. Ortiz (2005), en su estudio sobre las ATAL, llega a advertir que esta modalidad de enseñanza de la lengua vehicular puede estar siendo utilizada como sistema de discriminación. García et al. (2008) concluyen que la segregación por especificidad aboca a la exclusión por guetización simbólica y alimenta cierta xenofobia 
institucional.

- Lo anterior tiene mucho que ver con su enfoque compensatorio, según el cual el desconocimiento de la lengua vehicular supone un déficit (aludiendo a la teoría del déficit de Bereiter y Engelman, 1966). En consecuencia, lo más urgente es el tratamiento especializado para paliar dicho déficit, ocasionando que la mayoría del profesorado no se considere responsable de la educación de este alumnado hasta que no domine la lengua vehicular (García Fernández y Moreno, 2002; García Fernández et al., 2009, 2010, 2011; García Medina, 2015), lo que constituye un claro indicio de escasa integración en el funcionamiento del centro. El déficit atribuido al alumnado extranjero y alófono permite, a ojos de la cultura hegemónica que impregna el currículum oficial, justificar la asimilación cultural.

- La crítica es unánime al referirse a la inadecuación de las aulas cerradas para enseñar la lengua vehicular. Son muchas y variadas las objeciones:

- $\mathrm{Al}$ aislarlo en aulas específicas, se priva al alumnado alófono de una mayor interacción con el alumnado autóctono, desaprovechando uno de los principales mecanismos de progreso lingǘstico (Sierra y Lasagabaster, 2005; Siqués, Perera y Vila, 2012).

- No responden a su objetivo de facilitar el dominio de la lengua vehicular para que el alumnado alófono se incorpore con garantías al aula ordinaria. Pues, como demuestran numerosas investigaciones (véase Cummins, 2001), para adquirir un dominio académico de la lengua, más formal y descontextualizado, se necesitan al menos 5 años.

- Se adopta un modelo de bilingüismo sustractivo que desatiende el desarrollo y no aprovecha el potencial de las lenguas maternas a la hora de aprender una segunda lengua -hipótesis de la interdependencia lingüística- (Cummins, 1979, 2001, 2002).

- Se antepone el dominio de la lengua vehicular a cualquier otro aprendizaje curricular e, incluso, a la socialización e inclusión del alumnado. Olvidando que el alumnado tiene que familiarizarse con el universo cultural que circunda a la lengua (Carrasco, 2001, 2003; Vila, 2002; García et al., 2008; García et al., 2009, 2010, 2011).

- Resulta contradictorio que, tratándose de un dispositivo altamente especializado, no se garantice una formación rigurosa del profesorado para la enseñanza de una segunda lengua. En muchos casos parece bastar con la buena disposición del profesorado (las Aulas de Enlace son un claro ejemplo).

Como ya se ha mencionado, Siqués, Perera y Vila (2012) defienden la utilización de las aulas abiertas, la modalidad que se pretendió implantar en Cataluña a partir de 2004 al proponer las Aulas de Acogida. Tal y como explican, la implantación de aulas abiertas se ha ido generalizando en todo el mundo (desde Baviera hasta Toronto), con algunas diferencias en cuanto al régimen de asistencia a clases específicas para el aprendizaje de la lengua vehicular; que va desde los casos en los que se fijan unas pocas horas semanales (combinándolo con apoyos en el aula ordinaria), hasta aquellos en los que el apoyo lingüístico se ofrece íntegramente en el aula ordinaria.

Según sus investigaciones y las distintas evaluaciones de las Aulas de Acogida en las que han participado (Vila, Perera y Serra, 2006; Vila et al., 2007 y 2009; Vila et al., 2008), las aulas abiertas muestran mayor eficacia a la hora de asegurar el aprendizaje de la lengua vehicular que las aulas cerradas. Aprovechando la diversidad de modalidades que las Aulas de Acogida adoptan en Cataluña (dada la flexibilidad que la normativa concede a los centros), los autores analizan distintas variables para evaluar su incidencia en el aprendizaje del catalán. En sus conclusiones, señalan que de entre todas las variables estudiadas -adaptación escolar, permanencia en el aula de acogida, edad, escolarización previa, lengua familiar y tiempo de residencia-, es la adaptación escolar la que influye de manera más significativa en el dominio de la lengua vehicular alcanzado por el alumnado extranjero. Es decir, se consiguen mejores resultados en el aprendizaje de la lengua vehicular cuando este se produce dentro del aula ordinaria. También, el análisis de la variable relativa a la permanencia en el aula de acogida viene a confirmar lo anterior, ya que los resultados indican que el alumnado extranjero tiene más posibilidades de desarrollar habilidades en catalán, como usuario básico, cuantas menos horas semanales pasa en el aula de acogida. No obstante, esto no significa 
que sea suficiente la interacción social para garantizar el aprendizaje de la lengua vehicular, en especial si hablamos de un dominio académico de la lengua, sino que será necesario un apoyo lingǘstico sistemático (sea dentro del aula ordinaria o en sesiones de apoyo complementarias y controladas).

Por su parte, Besalú (2006) recuerda que no podemos quedarnos en la consideración del dispositivo, en sí, para enseñar la lengua vehicular. El verdadero valor reside en el hecho de que se trata, tan solo, de una medida más dentro de una propuesta global e integrada para todo el centro educativo, que no se impone una forma única de concretar las estrategias y prácticas para atender la diversidad cultural y lingüística. Los centros agradecen este tipo de iniciativas abiertas, flexibles y operativas, que además vienen acompañadas de los recursos y medios concretos necesarios para su desarrollo. Sin embargo, reconoce el autor que la realidad educativa siempre es mucho más compleja y menos lineal que los planes y orientaciones oficiales, lo que ocasiona que los resultados de la aplicación del Plan LIC no hayan sido los mismos en todos los centros educativos.

Aun reconociendo la decisiva aportación que han significado las Aulas de Acogida durante estos años, en Cataluña se están replanteando su continuidad, pues las diferentes evaluaciones ponen de relieve ciertos aspectos mejorables, como el escaso uso cotidiano que el alumnado extranjero hace del catalán. Tampoco parecen superados algunos inconvenientes asociados a la integración real de estos dispositivos en el funcionamiento diario de los centros educativos; posiblemente el hecho de que se siga hablando de "aulas" (asociadas a una estructura graduada y rígida) cuando se trata de organizar apoyos flexibles, abiertos e inclusivos no aporte más que cierta confusión entre el profesorado. Sin duda, hay que contar con la inercia que contribuye a perpetuar las clásicas relaciones verticales entre la administración y los centros, cuando se trata de procesos de innovación. En este orden de cosas la simple posibilidad, concedida por la administración, de que el centro pueda optar por utilizar un espacio apartado de las aulas ordinarias, reduciendo la participación en los procesos habituales de aprendizaje y de convivencia, debería ser revisada.

\section{Conclusiones}

Llegados a este punto, resulta pertinente mencionar las categóricas conclusiones del estudio encargado por el Comité Español de UNICEF a la Universidad Autónoma de Barcelona (Tarabini, 2017), que vienen a advertir sobre la ineficacia de las medidas de atención especial implantadas en nuestro sistema educativo. Además de implicar la clasificación y estigmatización del alumnado considerado problemático, en riesgo o excluido, no tienen incidencia sobre el conjunto del sistema educativo, en cuanto a la mejora de los resultados se refiere.

Se manifiesta en el mismo sentido González (2016), tras examinar los programas extraordinarios de atención a la diversidad que abundan en nuestro sistema educativo. Según sus conclusiones, en estos programas no se alteran los modelos tradicionales de enseñanza que, desafortunadamente, siguen muy presentes en las aulas ordinarias. Por otra parte, se trata de programas implantados por la administración sin que los propios centros lleguen a considerarlos como suyos. Finalmente, no se presta atención a las condiciones organizativas que son necesarias para desarrollar con garantías dichos programas, ni suele abordarse una revisión del proyecto educativo para integrarlos, en coherencia con la filosofía educativa del centro.

Las aulas de acogida lingüística no son una excepción. En su versión más rígida y cerrada suponen la segregación del alumnado alófono, con sus inevitables consecuencias asociadas a la modificación artificial de los espacios de convivencia, la alteración de los tiempos dedicados al aprendizaje y la desigualdad de oportunidades de acceso al conocimiento. La inclusión, ya se mencionó, se entiende como el proceso gracias al cual se asegura una mayor participación del alumnado (Ainscow, 2001), lo que resulta incompatible con la práctica de apartar a los y las estudiantes del desarrollo cotidiano de las sesiones de clase y de los espacios y momentos de convivencia espontáneos. Salvo en el caso de las Aulas de Acogida, tampoco se fomenta la implicación de todo el profesorado, ni de la comunidad educativa, en la atención del alumnado alófono; mientras que la revisión del currículo no está prevista. Por tanto, se puede afirmar que 
estas aulas no constituyen una medida inclusiva.

Si alguna duda quedara al respecto, el proyecto INCLUD-ED -Strategies for inclusion and social cohesion in Europe from education- (2011), aporta sobradas evidencias científicas de que la segregación del alumnado en distintos itinerarios únicamente genera desigualdades. Sin embargo, los agrupamientos basados en criterios de inclusión educativa -reorganización de los recursos humanos existentes dentro del aula ordinaria para atender a grupos heterogéneos de estudiantesproporcionan igualdad de oportunidades tanto para el éxito académico como para la inclusión social.

En segundo lugar, y tomando como referencia lo que ya se adelantó en el segundo apartado de este artículo (Oliver, 2010), es evidente que las aulas de acogida lingüística tampoco pueden considerarse una modalidad de agrupamiento flexible. En la mayoría de las ocasiones su implantación no ha supuesto un cambio en la organización del centro, ni tan siquiera una reflexión por parte de los equipos docentes. No es habitual una reelaboración curricular ni una transformación de las metodologías didácticas para orientar la intervención educativa en estas aulas. Es improbable que se cuente con el deseo de participar de los implicados y, mucho menos, con el consenso de la comunidad educativa. No se ha realizado un análisis certero de las necesidades educativas del alumnado extranjero de incorporación tardía, como revelan numerosas investigaciones (véase el cuarto apartado de este artículo). Se parte de una concepción bastante dudosa de lo que implica la diversidad del alumnado, por lo que resulta imposible evitar que terminen dándose situaciones de segregación y marginación. Y aún se podría añadir que, en muchos de los casos, quienes toman las decisiones sobre la pertinencia y organización de este tipo de agrupamientos no son ni los profesores o profesoras ni los centros educativos, ¿̇cómo se garantiza entonces la adaptación de la enseñanza más acorde con las necesidades concretas del alumnado atendido?

En tercer y último lugar, la mayor parte de estas aulas no están integradas en el funcionamiento ni en la estructura del centro educativo (el caso de las Aulas de Enlace es significativo, como muestran Sánchez y García, 2011). En muchas comunidades autónomas surgieron como la respuesta más rápida y poco comprometida al desconcierto de parte del profesorado por el incremento de la diversidad cultural y lingüística, de manera que su percepción en los centros fue el de un dispositivo externo, ajeno al funcionamiento de la escuela, atendido por profesorado ocasional, especialistas en atención a los migrantes (Ortiz, 2006) y responsables únicos de este alumnado (García et al, 2009). Por ejemplo, muchas Aulas de Enlace se dispusieron en espacios retirados o descartados para la actividad académica, buena parte de las ATAL son itinerantes y el profesorado no pertenece al claustro, suele ser la administración (sin contar demasiado con los centros) la que toma la decisión sobre la implantación de estas aulas, etc. En definitiva, pequeñas muestras de que los centros educativos no han llegado a asumir, como suya, la tarea de atender las necesidades culturales y lingǘsticas del alumnado; los centros simplemente han accedido a mantener en sus instalaciones un dispositivo regulado por la administración y, aparentemente, autónomo y desvinculado del normal funcionamiento y de las decisiones pedagógicas que les conciernen.

Quizá pueda parecer exagerado hablar de desregulación, pero a la administración educativa no le queda más remedio que admitir lo que ya sabemos desde hace mucho tiempo: que la naturaleza compleja de la educación y la diversidad humana, en todas sus expresiones, convierten cada centro, cada aula, cada grupo de estudiantes o profesionales en realidades únicas que demandan una atención educativa ajustada a su naturaleza y necesidades. Nadie mejor, al reflexionar sobre la complejidad de la educación, que Morin:

"Nuestra educación nos enseña a vivir de forma muy parcial e insuficiente, y es que nuestra educación se aparta de la vida, ignorando los problemas permanentes del vivir que acabamos de mencionar y dividiendo los conocimientos en compartimentos estancos. La tendencia tecnoeconómica, cada vez más poderosa e influyente, tiende a reducir la educación a la adquisición de competencias socioprofesionales en detrimento de las competencias existenciales, que pueden regenerar la cultura e 
introducir temas vitales en la enseñanza.” $(2016,26)$

Sin duda, este tipo de aulas, en la mayoría de sus manifestaciones, constituyen un claro ejemplo de la parcelación del conocimiento, de su desarraigo de aquellos contextos de la vida en los que cobra sentido y de la despreocupación por el desarrollo de competencias existenciales. Si algo han demostrado con meridiana claridad, es que estas aulas aportan muy poco al objetivo principal de educar para la vida, que defiende Morin.

\section{Referencias}

Aguadez, E.M. (2005). Diagnóstico basado en el currículum intercultural de aulas multiculturales en educación obligatoria (Tesis Doctoral). Universidad de Granada.

Ainscow, M. (2001). Desarrollo de escuelas inclusivas. Ideas, propuestas y experiencias para mejorar las instituciones escolares. Madrid: Narcea.

Antúnez, S., \& Gairín, J. (1994). La organización escolar. Práctica y fundamentos. Barcelona: Graó.

Arroyo, M.J. (2014). Aprendiraje de la lengua y alumnado inmigrante: un acercamiento a las aulas de adaptación lingüistica y social. Aulas ALISO. Madrid: Ministerio de Educación, Cultura y Deporte (Estudios CREADE).

Bereiter, C., \& Engelman, J. (1966). Teaching disadvantage children in the Preschool. New Jersey: PrenticeHall.

Besalú, X. (2006). El plan para la lengua y la cohesión social (Plan LIC) del gobierno de Cataluña. Revista Interuniversitaria de Formación del Profesorado, 20(2), pp. 45-68.

Bonal, X. (2004). Escola. La inmigració a debat: diversitat i ordenament jurídic. Debats, 3, pp. 7-17.

Carrasco, S. (2001). La llengua en les relacions interculturals a l'escola. Revista de Didáctica de la Llengua i de la Literatura, 23, pp. 29-40.

Carrasco, S. (2003). La escolarización de los hijos e hijas de inmigrantes y de minorías étnicoculturales. Revista de Educación, 330, pp. 99-136.

Castilla, J. (2011). Las ATAL: una experiencia andaluza de atención al alumnado de nueva incorporación de origen extranjero. En García Castaño, F.J. y Kressova, N. (Coords.), Actas del I Congreso Internacional sobre Migraciones en Andalucia, (pp. 503-512). Granada: Instituto de Migraciones.

Comunidad de Madrid (2008). Instrucciones de 28 de julio de 2008 de la Viceconsejería de Educación de la Comunidad de Madrid por las que se regulan la escolarización y las aulas de enlace para los alumnos procedentes de sistemas educativos extranjeros. Recuperado el 10 de enero de 2018 de http://www.madrid.org/dat_capital/bienvenida/impresos_pdf/Instrucciones_ae_0809.pdf.

Consejería de Educación de la Junta de Andalucía (2007). ORDEN de 15 de enero de 2007, por la que se regulan las medidas y actuaciones a desarrollar para la atención del alumnado inmigrante y, especialmente, las Aulas Temporales de Adaptación Lingüistica. Sevilla: Junta de Andalucía. Recuperado de http://www.juntadeandalucia.es/boja/2007/33/d1.pdf.

Cummins, J. (1979). Linguistic interdependence and the educational development of bilingual children. Review of Educational Research, 49(2), pp. 222-251. doi: 10.3102/00346543049002222

Cummins, J. (2001). ¿Qué sabemos de la educación bilingüe? Perspectivas psicolingüísticas y sociológicas. Revista de Educación, 326, 37-61.

Cummins, J. (2002). Lenguaje, poder y pedagogía. Niños y niñas bilingües entre dos fuegos. Madrid: MEC/Morata.

Del Olmo, M. (2012). Buenas prácticas, ¿desde el punto de vista de quién? Una contribución a la controversia sobre las aulas de enlace. Revista de Educación, 358, pp. 111-128. doi: 10-4438/1988592X-RE-2012-358-185

Departament d'Educació, Generalitat de Catalunya (2004). Pla per a la Llengua i la Cohesió Social. Barcelona: Generalitat de Catalunya. Recuperado el 14 de septiembre de 2017 de: http://aulaintercultural.org/ca/2005/02/04/pla-per-a-la-llengua-i-la-cohesio-social/.

Echeita, G., \& Cuevas, I. (2011). La educación inclusiva. En Martín, E., \& Mauri, T. (Coords.), Orientación educativa. Atención a la diversidady educación inclusiva (pp. 11-28). Barcelona: Graó/MEC.

El Diari de l'Educació (21 de enero de 2015). Ensenyament canvia les aules d'acollida per aules d'integració social. Recuperado el 17 de septiembre de 2017 de de http://diarieducacio.cat/ensenyamentcanvia-les-aules-dacollida-per-aules-dintegracio-social/. 
Escudero, J.M. (Comp.) (2016). Inclusión y exclusión educativa: Realidades, miradas y propuestas. Valencia: Nau Llibres.

Fernández, J., \& García, F.J. (2015). El desarrollo normativo que regula las aulas escolares de nacionalidad extranjera. Profesorado. Revista de Curriculum y Formación del Profesorado, 19(1), pp. 468-495. Recuperado el 18 de septiembre de: http://www.ugr.es/\%7Erecfpro/rev191COL11.pdf.

Franzé, A. (2008). Diversidad cultural en la escuela. Algunas contribuciones antropológicas. Revista de Educación, 345, pp. 111-132.

García, F.J., Rubio, M., \& Bouachra, O. (2008). Población inmigrante y escuela en España: Un balance de investigación. En García Roca, J., \& Lacomba, J. (Eds.). La inmigración en la sociedad española (pp. 403-473). Barcelona: Bellaterra.

García, F. J., Olmos, A., \& Ouafaa, B. O. (2015). Inmigración, crisis y escuela. Migraciones, 37, pp. 239-263. doi: 10.14422/mig.i37.y2015.011.

García, J. A. y Moreno, I. (2002). La respuesta a las necesidades educativas de los hijos de inmigrantes en la Comunidad de Madrid. Madrid: Consejo Económico y Social.

García, J.A., Moreno, I., Sánchez, P., García, R., Goenechea, C., Cluse, C., Plancarte, I., \& González, P. (2009). Las aulas de enlace a examen. ¿Espacios de oportunidad o de segregación? Madrid: CERSA.

García Fernández, J.A., Sánchez, P., Moreno, I, \& Goenechea, C. (2010). Estudio del sistema y funcionamiento de las aulas de enlace de la Comunidad de Madrid. De la normativa institucional a la realidad cotidiana. Revista de Educación, 352, pp. 473-493.

García, J.A., Moreno, I., Sánchez, P., García, R., Goenechea, C., Cluse, C., Plancarte, I., \& González, P. (2011). Estudio del sistema y funcionamiento de las aulas de enlace madrileñas. Informe de investigación. En García Castaño, F.J., \& Carrasco, S. (Coords.), Población inmigrante y escuela: conocimientos y saberes de investigación (pp. 551-584). Madrid: IFIIE.

García Fernández, J.A., \& Moreno Herrero, I. (Coords.) (2014). Escuela, diversidad cultural e inclusión. Madrid: La Catarata.

García, R., Moreno, I., \& Salguero, J.M. (2014). Tercera Parte: Diversidad cultural e inclusión. En García, J.A., \& Moreno, I. (Coords.) Escuela, diversidad cultural e inclusión. Madrid: La Catarata.

García, R. (2015). Implantación irregular y decadencia de las aulas de enlace en la Comunidad de Madrid: un modelo lingüístico agotado. En García, F.J., Megías, A., \& Ortega, J. (Eds.) Actas del VIII Congreso sobre Migraciones Internacionales en España (p. 172). Granada: Instituto de Migraciones, Universidad de Granada.

Gibson, M., \& Carrasco, S. (2009). The Education of Immigrant Youth: Some Lessons From the U.S. and Spain. Theory Into Practice, 48(4), pp. 249-257. doi: 10.1080/00405840903188118.

Goenechea, C., García, J.A., \& Jiménez, R. A. (2011). Los dilemas de la atención educativa a los alumnos inmigrantes recién llegados. Estudio comparativo de los modelos andaluz (ATAL) y madrileño (Aulas de Enlace). Profesorado. Revista de Currículum y Formación de Profesorado, 15(3), pp. 263-278.

González, M.T. (2016). Una mirada al pasado más reciente: El fracaso escolar y los intentos de acometerlo a base de programas extraordinarios de atención a la diversidad. En Escudero, J.M. (Comp.) Inclusión y exclusión educativa: Realidades, miradas y propuestas. Valencia: Nau Llibres

Grañeras, M., Vázquez, E., Parra, A., Rodríguez, F., Madrigal, A., Vale, P., \& Mata, P. (2007). La atención lingüística al alumnado extranjero en el sistema educativo español: normativa, actuaciones y medidas. Revista de Educación, 343, pp. 149-174.

Iglesias, C., \& Goenechea, C. (2016). La itinerancia como rasgo distintivo del profesorado de las ATAL de Cádiz. Actas del CIMIE16 de AMIE. Recuperado el 18 de septiembre de 2017 de: http://amieedu.org/actascimie16/.

INCLUD-ED Consortium (2011). Actuaciones de éxito en las escuelas europeas. Madrid: IFIIE-Creade.

Inglés, M.D. (2009). Aulas de enlace: estudio de la implantación de un programa educativo experimental para recién llegados en la Comunidad de Madrid. ELUA. Estudios de Lingüistica, 23, pp. 135-160. doi: 10.14198/ELUA2009.23.07.

Jiménez, R.A. (2004). Inmigración, interculturalidad y currículo. La educación en una sociedad multicultural. Sevilla: Publicaciones MCEP

Jiménez, R.A. (Coord.) (2009). Estudio de la atención educativa prestada al alumnado inmigrante en las Aulas Temporales de Adaptación Lingüistica en Cádir. Informe de Investigación. Recuperado el 20 de septiembre de 2017 de: 
http://minerva.uca.es/publicaciones/asp/docs/tesis/cotrina $\% 20$ garc $\% \mathrm{C} 3 \% \mathrm{ADa} \% 20 \mathrm{y} \% 20$ otro s.pdf.

Martín, L., \& Mijares, L. (Eds.) (2007). Voces del aula. Madrid: Estudios CREADE.

Mc Andrew, M. (2001). Immigration et diversité à l'école. Montréal: Les Presses de l'Université de Montréal.

Molina, S. (1995). El alumnado. En Lorenzo, M., \& Sáenz, O. (Coord.). Organización escolar. Una perspectiva ecológica (pp. 91-114). Alcoy: Marfil.

Morin, E. (2016). Enseñar a vivir. Manifiesto para cambiar la educación. Barcelona: Paidós.

Navarro, J.L. (2003). Inmigración en España y conocimiento de la lengua castellana. El caso de los escolares inmigrados en Aragón. (Tesis doctoral), Universidad de Lleida.

Núñez, E., \& Cerrillo, M.R. (2004). Aulas de Enlace: una iniciativa pedagógica, integradora e intercultural. Tendencias Pedagógicas, 9, 203-215.

Oliver, C. (2010). Estrategias para la diversidad. Agrupamientos flexibles de alumnos. En de la Torre, S. (Dir.), Estrategias didácticas en el aula. Buscando la calidad y la innovación (pp. 161-176). Madrid: UNED.

ORDEN de 15 de enero de 2007, por la que se regulan las medidas y actuaciones a desarrollar para la atención del alumnado inmigrante $y$, especialmente, las Aulas Temporales de Adaptación Lingüistica. (BOJA de/02/2007).

Ortiz, M. (2005). Enseñanza de la lengua en contextos escolares de acogida. Educación y futuro digital. Recuperado el 20 de septiembre de 2017 de https://www.researchgate.net/publication/228431759_ensenanza_de_la_lengua_en_contextos _escolares_de_acogida.

Quicios, M.P., \& Miranda, I. (2005). La educación inicial de los hijos de los inmigrantes en las "aulas de enlace". Revista Electrónica de Investigación Educativa, 7(2). Recuperado el 21 de septiembre de 2017 de http://redie.uabc.mx/vol7no2/contenido-quicios.html.

Roca, E., Úcar, X., \& Massot, M. (2002). Programas y experiencias de educación con inmigrantes. En Gervilla, E. (Coord.), Globalización, inmigración y educación, (pp. 205-291). Granada: Diputación de Granada/Universidad de Granada.

Sánchez, P., \& García Medina, R. (2011). Organización de las aulas de enlace en la Comunidad de Madrid. Cultura y Educación, 23(1), pp. 129-139. doi: 10.1174/113564011794728614.

Sierra, J.M. y Lasagabaster, D. (2005). ¿Qué ocurre en las aulas multilingües y multiculturales? ¿Cómo se puede sacar el máximo provecho de esta experiencia educativa? En Lasagabaster, D. y Sierra Plo, J.M. (Eds.), Multilingüismo, competencia lingüistica y nuevas tecnologías (pp. 7-28). Barcelona: Horsori.

Siqués, C., Perera, S., \& Vila, I. (2012). Variables implicadas en la adquisición del catalán en las aulas de acogida de educación primaria de Cataluña. Un estudio empírico. Anales de psicología, 28(2), pp. 444-456.

Tarabini, A. (Dir.) (2017). Los factores de la exclusión educativa en España: Mecanismos, perfiles y espacios de intervención. Madrid: UNICEF Comité Español. Recuperado el 22 de septiembre de 2018 de: https://www.unicef.es/sites/unicef.es/files/comunicacion/Factores_de_exclusion_educativa_e n_espana.pdf.

Vila, I., Perera, S., \& Serra Bonet, J.M. (2006). Les Aules d'acollida en l'educació primària. Algunes dades del curs 2004-2005. Caixa d'Eines, 4, pp. 40-55.

Vila, I., Canal, I., Mayans, P., Perera, S., Serra, J.M., \& Siqués, C. (2007). Les aules d'acollida de l'educació primària i secundària obligatòria de Catalunya: un estudi comparatiu. Catalan Revien, 21(1), pp. 351-380.

Vila, I., Oller, J., Perera, S., Serra, J.M., \& Siqués, C. (2008). Lengua inicial y adquisición del catalán en las aulas de acogida. En Díez-Itza, E. (Ed.), Estudios de Desarrollo del Lenguaje y Educación (pp. 199-210). Oviedo: ICE de la Universidad de Oviedo.

Vila, I., Canal, I., Mayans, P., Perera, S., Serra, J.M., \& Siqués, C. (2009). Las aulas de acogida de la educación primaria de Cataluña el curso 2005-2006: sus efectos sobre el conocimiento de catalán y la adaptación escolar. Infancia y Aprendizaje, 32(3), pp. 307-327. doi: 10.1174/021037009788964123.

Vila, I. (2002). Reflexiones sobre la interculturalidad. Revista Mugak, 21, pp. 7-14. 